Research Paper

\title{
Association between CTLA-4 gene polymorphism and risk of rheumatoid arthritis: a meta-analysis
}

\author{
Chuankun Zhou ${ }^{1}$, Shutao Gao ${ }^{2}, \mathrm{Xi} \mathrm{Yuan}^{1}$, Zixing $\mathrm{Shu}^{1}$, Song $\mathrm{Li}^{1}$, Xuying Sun ${ }^{1}$, Jun Xiao ${ }^{1}$, Hui Liu ${ }^{3}$ \\ ${ }^{1}$ Department of Orthopedics, Tongji Hospital, Tongji Medical College, Huazhong University of Science and \\ Technology, Wuhan 430030, Hubei, China \\ ${ }^{2}$ Department of Spine Surgery, The First Affiliate Hospital of Xinjiang Medical University, Urumqi 830054, Xinjiang, \\ China \\ ${ }^{3}$ Department of Orthopedics Trauma and Microsurgery, Zhongnan Hospital, Wuhan University, Wuhan 430000, \\ Hubei, China
}

Correspondence to: Jun Xiao, Hui Liu; email: jun_xiao@hust.edu.cn, hui_liu@whu.edu.cn Keywords: CTLA-4, polymorphism, rheumatoid arthritis, meta-analysis

Received: December 10, $2020 \quad$ Accepted: June 19, $2021 \quad$ Published: August 2, 2021

Copyright: ( $) 2021$ Zhou et al. This is an open access article distributed under the terms of the Creative Commons Attribution License (CC BY 3.0), which permits unrestricted use, distribution, and reproduction in any medium, provided the original author and source are credited.

\section{ABSTRACT}

Cytotoxic T lymphocyte-associated protein 4 (CTLA-4) gene polymorphisms may be involved in the risk of Rheumatoid arthritis (RA). However, evidence for the association remains controversial. Therefore, we performed a meta-analysis to confirm the relationship between CTLA-4 gene polymorphisms and RA. The pooled odds ratios (ORs) and $95 \%$ confidence intervals (Cls) were calculated to assess the strength of association. Stratified analysis was conducted by ethnicity. In total, 66 case-control studies including 21681 cases and 23457 controls were obtained. For rs3087243 polymorphism, significant association was detected in Asians ( $A$ vs. $G$ : $O R=0.77,95 \% \mathrm{Cl}=0.65-0.90, P=0.001 ; A A$ vs. $G G: O R=0.67,95 \% \mathrm{Cl}=0.48-0.94, P=0.02$ ) and Caucasians ( $A$ vs. $G$ : $O R=0.89,95 \% \mathrm{Cl}=0.86-0.93, P<0.00001 ; A A$ vs. $G G: O R=0.81,95 \% C l=0.75-0.88, P<0.00001)$. For rs231775 polymorphism, significant association was observed in the overall ( $G$ vs. $A: O R=1.16,95 \% \mathrm{Cl}=1.08$ 1.25, $P<0.0001$; $G G$ vs. $A A$ : $O R=1.29,95 \% \mathrm{Cl}=1.12-1.50, P=0.0006)$, and in Asians ( $G$ vs. $A: O R=1.27,95 \% \mathrm{Cl}=1.10-$ 1.47, $P=0.001$; $G G$ vs. $A A$ : $O R=1.58,95 \% \mathrm{Cl}=1.24-2.01, P=0.0002)$, but not in Caucasians. However, there was no association between rs5742909 polymorphism and RA. This meta-analysis confirmed that rs3087243 and rs231775 polymorphism were associated with the risk of RA in both overall population and ethnic-specific analysis, but there was no association between rs 5742909 polymorphism and RA risk.

\section{INTRODUCTION}

Rheumatoid arthritis, one of the most common inflammatory joint diseases in humans, is characterized by inflammation in synovium, destruction of cartilage and bone, generation of autoantibody, and complications of systemic organs [1]. Although RA affects $0.5-1 \%$ of the Western populations, the worldwide incidence of RA is increasing with the aging trend of the population [2]. Because of the results of reduced physical function, declined work capacity, decreased quality of life, and increased comorbid risk, RA carries heavy socioeconomic burden [3]. RA is believed to be a consequence of both genetic factors and environmental factors though main etiology has not yet been clearly clarified. In twin studies $50-65 \%$ of the risk for developing RA is ascribed to its heritability [4], indicating genetic factors have a strong effect on RA. So far more than one hundred gene loci associated with RA risk have been identified by single nucleotide polymorphisms (SNPs) [5, 6]. Apart from the human leukocyte antigen (HLA) locus, a well-known genetic 
risk factor for RA, numbers of other susceptibility genes and loci have been characterized [6]. Recently, a growing body of non-HLA genetic predisposition studies have been conducted on the association with the risk of RA [7-9].

Cytotoxic T lymphocyte-associated protein 4 (CTLA-4), one of widely studied non-HLA susceptibility gene of RA, is mainly expressed on the surface of Treg cells and conventional $\mathrm{T}$ cells and suppresses self-reactive $\mathrm{T}$ cell responses via downregulating ligand availability for the costimulatory receptor CD28 to elicit inhibitory signals $[10,11]$. Besides, the polymorphisms of CTLA-4 have already been proved to be candidates of the risk of the common autoimmune diseases at the genetic level [12-15]. As RA is a $\mathrm{T}$ cell mediated autoimmune disorder and CTLA-4 plays a vital role in regulating $\mathrm{T}$ cell function $[11,12,16]$, it suggests that $C T L A-4$ expression or function is most likely associated with the pathogenesis of RA. Single nucleotide polymorphisms in the CTLA-4 gene may contribute to abnormal levels of $C T L A-4$, and subsequently play a leading part in the susceptibility to RA $[12,17,18]$.

Among the identified SNPs in this gene, these three loci of CTLA-4, +49A/G (rs231775), $-318 C / T$ (rs5742909) and CT60 G/A(rs3087243), are most-often studied for the association with the predisposition of RA [18-20]. However, the conclusions which previous reports drew are inconsistent and incomprehensive. Although the association of CTLA-4 genetic polymorphisms and the risk of RA has been assessed in several meta-analyses [21-23], some recent studies also described this association in different populations in the past several years $[9,15,24-27]$. Hence these studies should be included to increase statistical power and obtain the reliable conclusion. On the other hand, all the three common loci should be included to embody the association comprehensively while the previous meta-analysis only researched one or two of the above loci. In view of these, it is necessary to incorporate the latest research into investigating the association of the three polymorphisms of CTLA-4 with susceptibility to RA. Here we use the latest casecontrol data to carry out an updated and comprehensive meta-analysis and obtain a more accurate estimation of the effect of the 3 SNPs $(+49 A / G$ (rs231775), CT60 $G / A(\mathrm{rs} 3087243)$ and $-318 \quad C / T \quad($ rs5742909)) on $\mathrm{RA}$ risk.

\section{RESULTS}

\section{Characteristics of the studies}

Based on the predetermined inclusion criteria, 66 eligible case-control studies with 42 articles were enrolled ultimately in the current analysis $[8,9,13-15$, 17-20, 24-56]. These publications had a high methodological quality whose NOS stars were more than 6 in general. There were 22 studies with 16394 patients and 17453 controls for rs3087243 SNP [8, 9, $13-15,18,19,26,40,41,43,46-49,52,53,56], 34$ studies with 11452 patients and 12444 controls for rs231775 SNP [9, 14, 17, 19, 20, 24, 25, 28-39, 41-45, 49-51, 54], and 10 studies with 2477 patients and 2941 controls for rs5742909 SNP [14, 20, 27, 29, 34, 37-39, $44,56]$. The references of all enrolled articles were subject to scrutiny and no more ones were available. The process of study selection according to the PRISMA principle was generalized in Figure 1. Quality assessment of included studies was shown in Supplementary Table 1. Details of included studies were listed in Table 1. Allele/genotype frequencies were displayed in Table 2.

\section{Efficiency analysis}

\section{Meta-analysis of CTLA-4 CT60(rs3087243) SNP and RA susceptibility}

By analyzing quantitatively allele or genotype distribution of 16394 patients and 17453 controls, a significant association between RA and CTLA-4 CT60(rs3087243) SNP was observed in all genetic comparisons $(A$ vs. $G$ : $\mathrm{OR}=0.87,95 \% \mathrm{CI}=0.83$ $0.91, P<0.00001 ; A A$ vs. $G G$ : $\mathrm{OR}=0.80,95 \% \mathrm{CI}$ $=0.74-0.87, P<0.00001 ; A G$ vs. $A A: \mathrm{OR}=0.85,95 \%$ $\mathrm{CI}=0.80-0.90, P<0.0001 ; A A+A G$ vs. $G G$ : OR $=0.83,95 \% \mathrm{CI}=0.77-0.90, P<0.0001$, and $A A$ vs. $A G+$ $G G:$ OR $=0.88,95 \%$ CI $=0.83-0.94, P=0.0003$ ) (Table 3 and Figure 2). Among the 22 included studies, 17 studies were performed in Caucasians, 3 were in Asians, 1 was African and 1 was in Latin Americans. Likewise, we carried out a stratified analysis by race to evaluate the ethnicity effects. In Caucasians, a protective role of rs3087243 SNP on RA was detected in all the five genetic comparisons. Similarly, a decreased risk of RA was found among Asians in the allelic comparison $(\mathrm{OR}=0.77,95 \% \mathrm{CI}=0.65-0.90$, $P=0.001)$ and the homozygote comparison (OR = $0.67,95 \% \mathrm{CI}=0.48-0.94, P=0.02)$. The heterozygote model and dominant model detected also this correlation in Latin Americans and the allelic comparison detected this correlation in Africans, but both the two populations needed more enrolled studies to elevate statistical power because this analysis currently included individually only one study. The outcomes were shown in Table 3. Collectively, Subgroup analyses revealed a significant protective association in Caucasians and Asians. When the $I^{2}>$ $50 \%$ and $P>0.1$, the Fix-effect model was used for the synthesis; otherwise, the Random-effect model was used. 
Meta-analysis of CTLA-4 +49A/G (rs231775) SNP and RA susceptibility

By quantitative analysis of allele or genotype distribution of 11452 patients and 12444 controls, there was a significant risk association between RA and $C T L A-4+49 A / G$ (rs231775) SNP. The overall pooled ORs of all the populations were as follows: $G$ vs. $A$ : OR $=1.16,95 \% \mathrm{CI}=1.08-1.25, P<0.0001 ; G G$ vs. $A A$ : OR $=1.29,95 \% \mathrm{CI}=1.12-1.50, P=0.0006 ; G A$ vs. $A A$ : $\mathrm{OR}$ $=1.19,95 \% \mathrm{CI}=1.07-1.32, P=0.001 ; G G+G A$ vs. $A A$ : $\mathrm{OR}=1.24,95 \% \mathrm{CI}=1.11-1.39, P=0.0001$ and $G G$ vs. $G A+A A:$ OR $=1.15,95 \%$ CI $=1.02-1.30, P=0.02$. The main results of overall analyses were shown in Table 3. 17 studies were conducted on Caucasians, 14 on Asians, 2 on Africans and 1 on Latin Americans. Subsequently, stratified analysis by ethnicity was conducted to get more clarifications. In the subgroup analysis, a significantly increased risk of RA was observed among the Asian population in all genetic comparisons except heterozygote comparison ( $G$ vs. $A$ : $\mathrm{OR}=1.27,95 \% \mathrm{CI}$ $=1.10-1.47, P=0.001 ; G G$ vs. $A A: \mathrm{OR}=1.58,95 \% \mathrm{CI}$ $=1.24-2.01, P=0.0002 ; G G+G A$ vs. $A A:$ OR $=1.33$, $95 \% \mathrm{CI}=1.17-1.51, P<0.0001 ; G G$ vs. $G A+A A: \mathrm{OR}=$ $1.15,95 \% \mathrm{CI}=1.02-1.30, P=0.02)$. In Latin American population, rs231775 SNP was a significant risk factor of RA, but it only included single study and the result might be incredible. Besides, no association of the rs231775 SNP with RA risk was found among the Caucasian population in all genetic comparisons when the Elshazli's study [24] was excluded because of its heterogeneity ( $G$ vs. $A$ : $\mathrm{OR}=1.07,95 \% \mathrm{CI}=0.99-1.15, P$ $=0.08 ; G G$ vs. $A A$ : $\mathrm{OR}=1.07,95 \% \mathrm{CI}=0.92-1.23$,

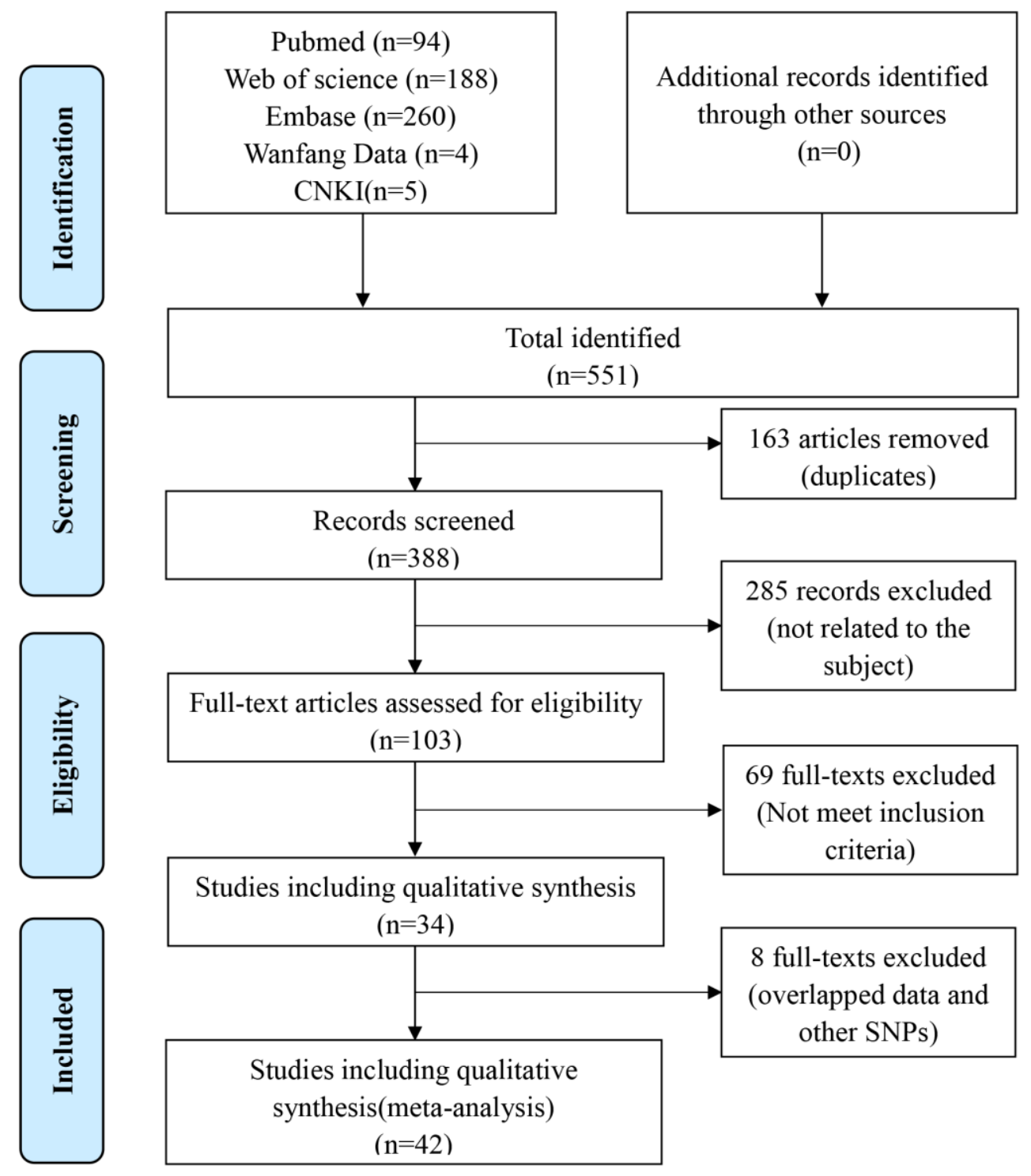

Figure 1. Flow diagram of the literature retrieval and screen. 
Table 1. Main characteristics of included studies.

\begin{tabular}{|c|c|c|c|c|c|c|c|c|}
\hline \multirow{2}{*}{ Study } & \multirow{2}{*}{ Year } & \multirow{2}{*}{ Country } & \multirow{2}{*}{ Ethnicity } & \multicolumn{2}{|c|}{ Numbers } & \multirow{2}{*}{$\begin{array}{c}\text { Genotype } \\
\text { method }\end{array}$} & \multirow{2}{*}{$\begin{array}{c}\text { Diagnostic } \\
\text { criteria }\end{array}$} & \multirow{2}{*}{$\begin{array}{c}\text { Quality } \\
\text { score }\end{array}$} \\
\hline & & & & $\mathbf{R A}$ & Con & & & \\
\hline \multicolumn{9}{|l|}{ Rs3087243(CT60) } \\
\hline Orozco & 2004 & Spain & Caucasian & 433 & 398 & TaqMan & ACR1987 & 7 \\
\hline Lei & 2005 & China & Asian & 326 & 250 & DGGE & ACR1987 & 8 \\
\hline Plenge (EIRA) & 2005 & Sweden & European & 1505 & 878 & MALDI-TOF & ACR1987 & 8 \\
\hline Plenge (NARAC) & 2005 & Sweden & European & 828 & 845 & MALDI-TOF & ACR1987 & 8 \\
\hline Zhernakova & 2005 & Dutch & Caucasian & 153 & 900 & PCR-RFLP & ACR1987 & 6 \\
\hline Suppiah & 2006 & Northern Ireland & Caucasian & 289 & 168 & PCR-RFLP & ACR1987 & 7 \\
\hline Costenbader & 2008 & USA & Caucasian & 423 & 420 & TaqMan & ACR1987 & 7 \\
\hline Tsukahara & 2008 & Japan & Asian & 1498 & 441 & TaqMan & ACR1987 & 8 \\
\hline Kelley & 2009 & USA & African & 505 & 712 & TaqMan & ACR1987 & 7 \\
\hline Daha & 2009 & Dutch & Caucasian & 867 & 863 & Sequenom & ACR1987 & 7 \\
\hline Barton & 2009 & UK & European & 3669 & 3049 & TaqMan & ACR1987 & 8 \\
\hline Walker & 2009 & Canada & Caucasian & 1140 & 1248 & Sequenom & ACR1987 & 8 \\
\hline Plant (1) & 2010 & France & Caucasian & 671 & 177 & Sequenom & ACR1987 & 8 \\
\hline Plant (2) & 2010 & Germany & Caucasian & 218 & 209 & Sequenom & ACR1987 & 8 \\
\hline Plant (3) & 2010 & Greece & Caucasian & 268 & 290 & Sequenom & ACR1987 & 8 \\
\hline Plant (4) & 2010 & UK & Caucasian & 1002 & 2725 & Sequenom & ACR1987 & 8 \\
\hline Danoy & 2011 & China & Asian & 1035 & 1702 & Sequenom & ACR1987 & 7 \\
\hline Torres-Carrillo & 2013 & Mexico & Latin American & 200 & 200 & PCR-RFLP & ACR1987 & 8 \\
\hline Luterek-Puszyńska & 2016 & Poland & Caucasian & 422 & 338 & TaqMan & ACR1987 & 7 \\
\hline Schulz & 2020 & Germany & Caucasian & 111 & 256 & PCR-RFLP & ACR2010 & 6 \\
\hline El-Gabalawy & 2011 & Canada & Caucasian & 332 & 490 & Sequenom & ACR1987 & 6 \\
\hline Vernerova & 2016 & Slovakia & Caucasian & 499 & 894 & TaqMan & ACR2010 & 9 \\
\hline \multicolumn{9}{|l|}{ Rs231775(49G/A) } \\
\hline AIFadhli & 2013 & Kuwait & Asian & 114 & 282 & PCR-RFLP & ACR1987 & 6 \\
\hline Barton (I) & 2000 & Spain & Caucasian & 136 & 144 & PCR-RFLP & ACR1987 & 7 \\
\hline Barton (II) & 2000 & UK & Caucasian & 192 & 96 & PCR-RFLP & ACR1987 & 7 \\
\hline Benhatchi & 2011 & Slovakia & Caucasian & 57 & 51 & PCR-RFLP & ACR1987 & 6 \\
\hline Elshazli & 2015 & Egypt & Caucasian & 112 & 122 & PCR-RFLP & ACR1987 & 6 \\
\hline Feng & 2005 & China & Asian & 50 & 60 & PCR-RFLP & ACR1987 & 6 \\
\hline Gonzalez-Escribano & 1999 & Spain & Caucasian & 138 & 305 & PCR-ARMS & ACR1987 & 6 \\
\hline Hadj & 2001 & Tunisia & African & 60 & 150 & PCR-RFLP & ACR1987 & 7 \\
\hline Lee 2002 & 2002 & Korea & Asian & 86 & 86 & PCR-RFLP & ACR1987 & 6 \\
\hline Lee 2003 & 2003 & China & Asian & 186 & 203 & PCR-RFLP & ACR1987 & 6 \\
\hline Lei & 2005 & China & Asian & 326 & 250 & DGGE & ACR1987 & 8 \\
\hline Liu 2004 & 2004 & Taiwan & Asian & 65 & 81 & PCR-RFLP & ACR1987 & 6 \\
\hline Barton & 2004 & UK & European & 132 & 156 & TaqMan & ACR1987 & 7 \\
\hline Liu 2013 & 2013 & China & Asian & 213 & 303 & PCR-RFLP & ACR1987 & 7 \\
\hline Luterek-Puszyńska & 2016 & Poland & Caucasian & 422 & 338 & TaqMan & ACR2010 & 7 \\
\hline Matsushita & 1999 & Japan & Asian & 461 & 150 & PCR-SSCP & ACR1987 & 7 \\
\hline Milicic & 2001 & UK & Caucasian & 421 & 452 & PCR-RFLP & ACR1987 & 8 \\
\hline Miterski & 2004 & Germany & Caucasian & 284 & 362 & PCR-RFLP & ACR1987 & 7 \\
\hline Munoz-Valle & 2010 & Mexico & Mexican & 199 & 199 & PCR-RFLP & ACR1987 & 6 \\
\hline Plant (1) & 2010 & France & Caucasian & 684 & 162 & Sequenom & ACR1987 & 8 \\
\hline Plant (2) & 2010 & Germany & European & 220 & 209 & Sequenom & ACR1987 & 8 \\
\hline Plant (3) & 2010 & Greece & European & 272 & 287 & Sequenom & ACR1987 & 8 \\
\hline Plant (4) & 2010 & UK & European & 1004 & 2659 & Sequenom & ACR1987 & 6 \\
\hline
\end{tabular}




\begin{tabular}{lcccccccc} 
Seidl & 1998 & Germany & Caucasian & 258 & 456 & RFLP-SSCP & ACR1987 & 8 \\
Suppiah & 2006 & UK & European & 289 & 475 & PCR-RFLP & ACR1987 & 7 \\
Takeuchi & 2006 & Japan & Asian & 100 & 104 & PCR-RFLP & ACR1987 & 6 \\
Tang & 2013 & China & Asian & 1489 & 1200 & TaqMan & ACR1987 & 8 \\
Tsukahara & 2008 & Japan & Asian & 1490 & 448 & TaqMan & ACR1987 & 8 \\
Kelley & 2009 & USA & African & 505 & 712 & TaqMan & ACR1987 & 7 \\
Vaidya & 2002 & UK & Caucasian & 123 & 349 & PCR-RFLP & ACR1987 & 6 \\
Walker & 2009 & Canada & Caucasian & 1140 & 1248 & Sequenom & ACR1987 & 8 \\
Yanagawa & 2000 & Japan & Asian & 85 & 200 & PCR-RFLP & ACR1987 & 6 \\
Zhou & 2007 & China & Asian & 39 & 44 & PCR-RFLP & ACR1987 & 6 \\
Sameem & 2015 & Pakistani & Asian & 100 & 100 & PCR-RFLP & RF test & 6 \\
Rs5742909 (318C/T) & & & & & & & \\
Gonzalez-Escribano & 1999 & Spain & Caucasian & 138 & 305 & PCR-ARMS & ACR1987 & 6 \\
Lee 2002 & 2002 & Korea & Asian & 86 & 86 & PCR-RFLP & ACR1987 & 6 \\
Barton & 2004 & UK & European & 151 & 152 & TaqMan & ACR1987 & 7 \\
Liu 2004 & 2004 & Tainan & Asian & 65 & 81 & PCR-RFLP & ACR1987 & 6 \\
Miterski & 2004 & Germany & Caucasian & 284 & 362 & PCR-RFLP & ACR1987 & 7 \\
Takeuchi & 2006 & Japan & Asian & 100 & 104 & PCR-RFLP & ACR1987 & 6 \\
Walker & 2009 & Canada & Caucasian & 1140 & 1248 & Sequenom & ACR1987 & 8 \\
Liu 2013 & 2013 & China & Asian & 213 & 303 & PCR-RFLP & ACR1987 & 7 \\
Torres-Carrillo & 2013 & Mexico & Latin American & 200 & 200 & PCR-RFLP & ACR1987 & 7 \\
Fattah & 2017 & Egypt & Caucasian & 100 & 100 & PCR-RFLP & ACR2010 & 6 \\
\hline
\end{tabular}

Table 2. Distribution of genotype and allele among RA patients and controls.

\begin{tabular}{|c|c|c|c|c|c|c|c|c|c|c|c|}
\hline \multirow{2}{*}{ Study } & \multicolumn{5}{|c|}{ Cases } & \multicolumn{5}{|c|}{ Controls } & \multirow{2}{*}{ HEW } \\
\hline & MM & Mm & $\mathbf{m m}$ & $\mathbf{M}$ & $\mathbf{m}$ & MM & Mm & $\mathbf{m m}$ & $\mathbf{M}$ & $\mathbf{m}$ & \\
\hline \multicolumn{12}{|l|}{ Rs3087243(CT60) } \\
\hline Orozco & 118 & 198 & 117 & 434 & 432 & 98 & 199 & 101 & 395 & 401 & YES \\
\hline Lei & 33 & 137 & 156 & 203 & 449 & 32 & 131 & 87 & 195 & 305 & YES \\
\hline Plenge (EIRA) & 230 & 680 & 595 & 1140 & 1870 & 145 & 396 & 337 & 686 & 1070 & YES \\
\hline Plenge (NARAC) & 133 & 387 & 308 & 653 & 1003 & 165 & 426 & 254 & 756 & 934 & YES \\
\hline Zhernakova & NA & NA & NA & 133 & 173 & NA & NA & NA & 841 & 959 & NA \\
\hline Suppiah & NA & NA & NA & 234 & 344 & NA & NA & NA & 145 & 191 & NA \\
\hline Costenbader & 82 & 201 & 140 & 365 & 481 & 87 & 195 & 138 & 369 & 471 & YES \\
\hline Tsukahara & 87 & 538 & 873 & 712 & 2284 & 33 & 163 & 245 & 229 & 653 & YES \\
\hline Kelley & $\mathrm{NA}$ & NA & NA & NA & 505 & NA & NA & NA & NA & 712 & NA \\
\hline Daha & NA & NA & NA & 729 & 1005 & NA & NA & NA & 785 & 941 & NA \\
\hline Barton & 677 & 1760 & 1232 & 3114 & 4224 & 634 & 1523 & 892 & 2791 & 3307 & YES \\
\hline Walker & 207 & 518 & 415 & 932 & 1348 & 273 & 613 & 362 & 1159 & 1337 & YES \\
\hline Plant (1) & 131 & 332 & 208 & 594 & 748 & 45 & 91 & 41 & 181 & 173 & YES \\
\hline Plant (2) & 35 & 105 & 78 & 175 & 261 & 35 & 101 & 73 & 171 & 247 & YES \\
\hline Plant (3) & 55 & 135 & 78 & 245 & 291 & 70 & 145 & 75 & 285 & 295 & YES \\
\hline Plant (4) & 204 & 487 & 311 & 895 & 1109 & 542 & 1344 & 839 & 2428 & 3022 & YES \\
\hline Danoy & NA & NA & NA & 310 & 1760 & NA & NA & NA & 681 & 2723 & NA \\
\hline Torres-Carrillo & 31 & 86 & 83 & 148 & 252 & 32 & 106 & 62 & 170 & 230 & YES \\
\hline Luterek-Puszyńska & 53 & 193 & 176 & 299 & 545 & 45 & 174 & 119 & 264 & 412 & YES \\
\hline Schulz & 13 & 49 & 49 & 75 & 147 & 42 & 124 & 90 & 208 & 304 & YES \\
\hline El-Gabalawy & 45 & 161 & 126 & 251 & 413 & 66 & 226 & 198 & 358 & 622 & YES \\
\hline
\end{tabular}




\begin{tabular}{|c|c|c|c|c|c|c|c|c|c|c|c|}
\hline Vernerova & NA & NA & NA & 616 & 382 & NA & NA & NA & 1064 & 1064 & NA \\
\hline \multicolumn{12}{|l|}{ Rs231775(49G/A) } \\
\hline AIFadhli & 10 & 30 & 74 & 50 & 178 & 14 & 86 & 182 & 114 & 450 & YES \\
\hline Barton (I) & 14 & 57 & 65 & 85 & 187 & 12 & 70 & 62 & 94 & 194 & YES \\
\hline Barton (II) & 38 & 86 & 68 & 162 & 222 & 19 & 51 & 26 & 89 & 103 & YES \\
\hline Benhatchi & 6 & 33 & 18 & 45 & 69 & 5 & 25 & 21 & 35 & 67 & YES \\
\hline Elshazli & 14 & 55 & 43 & 83 & 141 & 6 & 45 & 71 & 57 & 187 & YES \\
\hline Feng & 20 & 21 & 9 & 61 & 39 & 9 & 32 & 19 & 50 & 70 & YES \\
\hline Gonzalez-Escribano & 10 & 63 & 65 & 83 & 193 & 30 & 103 & 172 & 163 & 447 & NO \\
\hline Hadj & 23 & 27 & 10 & 73 & 47 & 68 & 62 & 20 & 198 & 102 & YES \\
\hline Lee 2002 & 41 & 35 & 10 & 117 & 55 & 49 & 29 & 8 & 127 & 45 & YES \\
\hline Lee 2003 & 103 & 67 & 16 & 273 & 99 & 85 & 100 & 18 & 270 & 136 & YES \\
\hline Lei & 148 & 138 & 40 & 434 & 218 & 86 & 125 & 39 & 297 & 203 & YES \\
\hline Liu 2004 & 14 & 42 & 9 & 70 & 60 & 21 & 50 & 10 & 92 & 70 & NO \\
\hline Barton & 34 & 55 & 43 & 123 & 141 & 29 & 68 & 59 & 126 & 186 & YES \\
\hline Liu 2013 & 77 & 111 & 25 & 265 & 161 & 130 & 125 & 48 & 385 & 221 & YES \\
\hline Luterek-Puszyńska & 79 & 210 & 133 & 368 & 476 & 63 & 160 & 115 & 286 & 390 & YES \\
\hline Matsushita & 200 & 199 & 62 & 599 & 323 & 56 & 72 & 22 & 184 & 116 & YES \\
\hline Milicic & 63 & 223 & 135 & 349 & 493 & 73 & 213 & 166 & 359 & 545 & YES \\
\hline Miterski & NA & NA & NA & 222 & 346 & NA & NA & NA & 269 & 455 & NA \\
\hline Munoz-Valle & 42 & 102 & 55 & 186 & 212 & 34 & 82 & 83 & 150 & 248 & YES \\
\hline Plant (1) & 96 & 315 & 273 & 507 & 861 & 15 & 75 & 72 & 105 & 219 & YES \\
\hline Plant (2) & 37 & 111 & 72 & 185 & 255 & 32 & 94 & 83 & 158 & 260 & YES \\
\hline Plant (3) & 26 & 133 & 113 & 185 & 359 & 33 & 107 & 147 & 173 & 401 & YES \\
\hline Plant (4) & 146 & 451 & 407 & 743 & 1265 & 410 & 1255 & 994 & 2075 & 3243 & YES \\
\hline Seidl & 37 & 138 & 83 & 212 & 304 & 68 & 210 & 179 & 346 & 568 & YES \\
\hline Suppiah & 40 & 144 & 105 & 224 & 354 & 92 & 241 & 142 & 425 & 525 & YES \\
\hline Takeuchi & 49 & 39 & 12 & 137 & 63 & 44 & 49 & 11 & 137 & 71 & YES \\
\hline Tang & 652 & 642 & 195 & 1946 & 1032 & 474 & 535 & 191 & 1483 & 917 & YES \\
\hline Tsukahara & 636 & 668 & 186 & 1940 & 1040 & 181 & 194 & 73 & 556 & 340 & YES \\
\hline Kelley & NA & NA & NA & NA & 505 & NA & NA & NA & NA & 712 & NA \\
\hline Vaidya & 20 & 65 & 38 & 105 & 141 & 45 & 158 & 146 & 248 & 450 & YES \\
\hline Walker & 177 & 554 & 409 & 908 & 1372 & 178 & 577 & 493 & 933 & 1563 & YES \\
\hline Yanagawa & 29 & 50 & 6 & 108 & 62 & 78 & 88 & 34 & 244 & 156 & YES \\
\hline Zhou & 22 & 9 & 8 & 53 & 25 & 8 & 14 & 22 & 30 & 58 & YES \\
\hline Sameem & 54 & 26 & 20 & 134 & 66 & 28 & 31 & 41 & 87 & 113 & NO \\
\hline \multicolumn{12}{|l|}{$\operatorname{Rs5742909}(318 C / T)$} \\
\hline Gonzalez-Escribano & 1 & 29 & 108 & 31 & 245 & 2 & 60 & 243 & 64 & 546 & NO \\
\hline Lee 2002 & 2 & 19 & 65 & 23 & 149 & 4 & 14 & 68 & 22 & 150 & YES \\
\hline Barton & 1 & 18 & 132 & 20 & 282 & 3 & 27 & 122 & 33 & 271 & YES \\
\hline Liu 2004 & 0 & 15 & 50 & 15 & 115 & 0 & 23 & 58 & 23 & 139 & NO \\
\hline Miterski & NA & NA & NA & 64 & 504 & NA & NA & NA & 50 & 674 & NA \\
\hline Takeuchi & 0 & 13 & 87 & 13 & 187 & 0 & 22 & 82 & 22 & 186 & YES \\
\hline Walker & 13 & 219 & 908 & 245 & 2035 & 10 & 183 & 1055 & 203 & 2293 & YES \\
\hline Liu 2013 & 14 & 97 & 102 & 125 & 301 & 13 & 77 & 213 & 103 & 503 & YES \\
\hline Torres-Carrillo & 2 & 16 & 182 & 20 & 380 & 0 & 20 & 180 & 20 & 380 & YES \\
\hline Fattah & 7 & 52 & 41 & 66 & 134 & 2 & 32 & 66 & 36 & 164 & YES \\
\hline
\end{tabular}

$M$, minor allele; m, major allele; NA, not available; HWE, Hardy-Weinberg Equilibrium. 
Table 3. Results of different comparative genetic models on the association of CTLA-4 SNPs with RA.

\begin{tabular}{|c|c|c|c|c|c|c|c|c|c|}
\hline \multirow{2}{*}{ Genetic model } & \multirow{2}{*}{ Population } & \multirow{2}{*}{ Cases } & \multirow{2}{*}{ Controls } & \multicolumn{3}{|c|}{ Association } & \multicolumn{3}{|c|}{ Heterogeneity } \\
\hline & & & & OR & $95 \% \mathrm{CI}$ & $P$-value & Model & $I^{2}$ & $P$-value \\
\hline \multicolumn{10}{|l|}{ Rs308724 } \\
\hline \multirow{5}{*}{ A vs. $\mathrm{G}$} & Total & 16394 & 17453 & 0.87 & $0.83-0.91$ & $<0.00001$ & REM & 39 & 0.003 \\
\hline & Caucasian & 12830 & 14148 & 0.89 & $0.86-0.93$ & $<0.00001$ & FEM & 25 & 0.17 \\
\hline & Asian & 2859 & 2393 & 0.77 & $0.65-0.90$ & 0.001 & REM & 56 & 0.10 \\
\hline & Latin & 200 & 200 & 0.79 & $0.60-1.06$ & 0.11 & - & - & - \\
\hline & African & 505 & 712 & 0.83 & $0.67-1.02$ & 0.08 & - & - & - \\
\hline \multirow{5}{*}{ AA vs. GG } & Total & 13046 & 12214 & 0.80 & $0.74-0.87$ & $<0.00001$ & FEM & 22 & 0.20 \\
\hline & Caucasian & 11022 & 11323 & 0.81 & $0.75-0.88$ & $<0.00001$ & FEM & 32 & 0.13 \\
\hline & Asian & 1824 & 691 & 0.67 & $0.48-0.94$ & 0.02 & FEM & 0 & 0.48 \\
\hline & Latin & 200 & 200 & 0.72 & $0.40-1.31$ & 0.29 & - & - & - \\
\hline & Total & 13046 & 12214 & 0.85 & $0.80-0.90$ & $<0.0001$ & FEM & 28 & 0.14 \\
\hline \multirow{3}{*}{ AG vs. GG } & Caucasian & 11022 & 11323 & 0.86 & $0.81-0.92$ & $<0.0001$ & FEM & 11 & 0.33 \\
\hline & Asian & 1824 & 691 & 0.75 & $0.48-1.18$ & 0.21 & REM & 78 & 0.03 \\
\hline & Latin & 200 & 200 & 0.61 & $0.39-0.94$ & 0.02 & - & - & - \\
\hline \multirow{5}{*}{$\mathrm{AA}+\mathrm{GA}$ vs. GG } & Total & 13046 & 12214 & 0.83 & $0.77-0.90$ & $<0.0001$ & REM & 46 & 0.02 \\
\hline & Caucasian & 11022 & 11323 & 0.85 & $0.78-0.93$ & $<0.0002$ & REM & 40 & 0.07 \\
\hline & Asian & 1824 & 691 & 0.74 & $0.48-1.12$ & 0.15 & REM & 77 & 0.04 \\
\hline & Latin & 200 & 200 & 0.60 & $0.40-0.90$ & 0.01 & - & - & - \\
\hline & Total & 13046 & 12214 & 0.88 & $0.83-0.94$ & 0.0003 & FEM & 0 & 0.75 \\
\hline \multirow{3}{*}{ AA vs. GA+GG } & Caucasian & 11022 & 11323 & 0.89 & $0.83-0.95$ & 0.0008 & FEM & 0 & 0.60 \\
\hline & Asian & 1824 & 691 & 0.76 & $0.55-1.06$ & 0.10 & FEM & 0 & 0.98 \\
\hline & Latin & 200 & 200 & 0.96 & $0.56-1.65$ & 0.89 & - & - & - \\
\hline \multicolumn{10}{|l|}{ Rs231775 } \\
\hline \multirow{5}{*}{ G vs. A } & Total & 11452 & 12444 & 1.16 & $1.08-1.25$ & $<0.0001$ & REM & 66 & 0.00001 \\
\hline & Caucasian & 5884 & 7872 & 1.09 & $1.01-1.19$ & 0.04 & REM & 38 & 0.004 \\
\hline & Asian & 4804 & 3511 & 1.27 & $1.10-1.47$ & 0.001 & REM & 71 & $<0.0001$ \\
\hline & African & 565 & 862 & 1.06 & $0.68-1.65$ & 0.81 & REM & 73 & 0.05 \\
\hline & Latin & 199 & 199 & 1.45 & $1.09-1.92$ & 0.010 & - & - & - \\
\hline \multirow{5}{*}{ GG vs. AA } & Total & 10663 & 11370 & 1.29 & $1.12-1.50$ & 0.0006 & REM & 54 & 0.0002 \\
\hline & Caucasian & 5600 & 7510 & 1.11 & $0.94-1.31$ & 0.21 & FEM & 25 & 0.17 \\
\hline & Asian & 4804 & 3511 & 1.58 & $1.24-2.01$ & 0.0002 & REM & 51 & 0.01 \\
\hline & African & 60 & 150 & 0.68 & $0.28-1.65$ & 0.39 & - & - & - \\
\hline & Latin & 199 & 199 & 1.24 & $1.09-1.42$ & 0.03 & - & - & - \\
\hline \multirow{5}{*}{ GA vs. AA } & Total & 10663 & 11370 & 1.19 & $1.07-1.32$ & 0.001 & REM & 46 & 0.003 \\
\hline & Caucasian & 5600 & 7510 & 1.18 & $1.02-1.35$ & 0.02 & REM & 59 & 0.001 \\
\hline & Asian & 4804 & 3511 & 1.20 & $1.05-1.38$ & 0.08 & FEM & 3 & 0.42 \\
\hline & African & 60 & 150 & 0.87 & $0.36-2.11$ & 0.76 & - & - & - \\
\hline & Latin & 199 & 199 & 1.88 & $1.20-2.94$ & 0.006 & - & - & - \\
\hline \multirow{5}{*}{ GG+GA vs. AA } & Total & 10663 & 11370 & 1.24 & $1.11-1.39$ & 0.0001 & FEM & 56 & 0.001 \\
\hline & Caucasian & 5600 & 7510 & 1.17 & $1.02-1.34$ & 0.02 & REM & 62 & 0.0006 \\
\hline & Asian & 4804 & 3511 & 1.33 & $1.17-1.51$ & $<0.0001$ & FEM & 31 & 0.12 \\
\hline & African & 60 & 150 & 0.77 & $0.34-1.76$ & 0.53 & - & - & - \\
\hline & Latin & 199 & 199 & 1.87 & $1.23-2.85$ & 0.003 & - & - & - \\
\hline \multirow{2}{*}{ GG vs. $\mathrm{GA}+\mathrm{AA}$} & Total & 10663 & 11370 & 1.15 & $1.02-1.30$ & 0.02 & REM & 57 & $<0.0001$ \\
\hline & Caucasian & 5600 & 7510 & 1.01 & $0.91-1.12$ & 0.80 & FEM & 10 & 0.34 \\
\hline
\end{tabular}




\begin{tabular}{lccccccccc}
\multirow{7}{*}{ Rs5742909 } & Asian & 4804 & 3511 & 1.34 & $1.08-1.65$ & 0.008 & REM & 72 & $<0.0001$ \\
& African & 60 & 150 & 0.75 & $0.41-1.38$ & 0.36 & - & - & - \\
\multirow{5}{*}{ T vs. C } & Latin & 199 & 199 & 1.30 & $0.79-2.15$ & 0.31 & - & - & - \\
& & & & & & & & & \\
& Total & 2477 & 2941 & 1.21 & $0.93-1.57$ & 0.15 & REM & 71 & 0.0003 \\
& Caucasian & 1813 & 2167 & 1.31 & $0.94-1.84$ & 0.11 & REM & 73 & 0.005 \\
& Asian & 464 & 574 & 1.05 & $0.56-1.96$ & 0.88 & REM & 80 & 0.002 \\
TT vs. CC & Latin & 200 & 200 & 1.00 & $0.53-1.89$ & 1.00 & - & - & - \\
& Total & 2193 & 2579 & 1.71 & $1.08-2.73$ & 0.08 & FEM & 17 & 0.30 \\
& Caucasian & 1529 & 1805 & 1.58 & $0.60-4.17$ & 0.35 & REM & 32 & 0.22 \\
& Asian & 464 & 574 & 1.34 & $0.34-5.28$ & 0.68 & REM & 56 & 0.13 \\
TC vs. CC & Latin & 200 & 200 & 4.95 & $0.24-103.73$ & 0.30 & - & - & - \\
& Total & 2193 & 2579 & 1.19 & $0.84-1.69$ & 0.33 & FEM & 76 & $<0.0001$ \\
& Caucasian & 1529 & 1805 & 1.27 & $0.81-1.99$ & 0.29 & REM & 74 & 0.01 \\
& Asian & 464 & 574 & 1.16 & $0.53-2.56$ & 0.70 & REM & 83 & 0.0004 \\
TT+TC vs. CC & Latin & 200 & 200 & 0.79 & $0.40-1.58$ & 0.51 & - & - & - \\
& Total & 2193 & 2579 & 1.19 & $0.84-1.69$ & 0.33 & FEM & 77 & $<0.0001$ \\
& Caucasian & 1529 & 1805 & 1.28 & $0.79-2.07$ & 0.32 & REM & 78 & 0.003 \\
& Asian & 464 & 574 & 1.12 & $0.52-2.43$ & 0.77 & REM & 84 & 0.0003 \\
& Latin & 200 & 200 & 0.89 & $0.46-1.74$ & 0.73 & - & - & - \\
TT vs. TC+CC & Total & 2193 & 2579 & 1.43 & $0.90-2.27$ & 0.13 & FEM & 0 & 0.52 \\
& Caucasian & 1529 & 1805 & 1.46 & $0.77-2.78$ & 0.25 & FEM & 0 & 0.39 \\
& Asian & 464 & 574 & 1.27 & $0.63-2.54$ & 0.51 & FEM & 32 & 0.23 \\
& Latin & 200 & 200 & 5.05 & $0.24-105.86$ & 0.30 & - & - & - \\
\hline
\end{tabular}

$\mathrm{OR}$, odds ratio; $\mathrm{Cl}$, confidence interval; FEM, fix-effect model; REM, random-effect model.

$P=0.37 ; G A$ vs. $A A$ : $\mathrm{OR}=1.15,95 \% \mathrm{CI}=1.00-1.31$, $P=0.05 ; G G+G A$ vs. $A A:$ OR $=1.14,95 \% \mathrm{CI}=1.00-$ $1.29, P=0.05$ and $G G$ vs. $G A+A A$ : $\mathrm{OR}=1.00,95 \%$ $\mathrm{CI}=0.90-1.11, P=0.98$ ) (Table 3 and Figure 3). There was no remarkable association between rs 231775 SNP and RA in Africans. The results were summarized in Table 3 and Figure 3. These data with moderate heterogeneity employed the random-effect model for the synthesis.

\section{Meta-analysis of CTLA-4 318C/T (rs5742909) SNP and $R A$ susceptibility}

Through the pooled analysis of genetic data of 2477 patients and 2941 controls in a total of 10 studies, of which 5 were conduct on Caucasians, 4 on Asians, and 1 on Latin Americans, no significant associations between rs5742909 SNP and RA in the overall pooled results were found among all populations for the allelic and genotypic comparisons ( $T$ vs. $C$ : OR $=1.21,95 \%$ CI $=0.93-1.57, P=0.15 ; T T$ vs. $C C:$ OR $=1.71,95 \%$ CI $=1.08-2.73, P=0.08 ; T C$ vs. $C C:$ OR $=1.19,95 \%$ CI $=0.84-1.69, P=0.33 ; T T+T C$ vs. $C C:$ OR $=1.19,95 \%$ $\mathrm{CI}=0.84-1.69, P=0.33$ and $T T$ vs. $T C+C C$ : $\mathrm{OR}=1.43$, 95\% CI=0.90-2.27, $P=0.13$ ) (Table 3 and Figure 4). Meanwhile, the subgroup analysis by ethnicity did not indicate any remarkable associations in all genetic models (Table 3 ). As the heterogeneity of genetic model existed, random effect model in this part was used to make a reliable result.

\section{Heterogeneity analysis and publication bias}

To ensure the reliability of the results, we first evaluate the heterogeneity (by $I^{2}$ ) and found that heterogeneity existed in some genetic models of rs231775 SNP and rs5742909 SNP (Table 3). In order to minimize heterogeneity, the following methods were carried out in this meta-analysis. On the one hand, the random-effect models were exploited in the genetic models with moderate heterogeneity $\left(I^{2}>50 \%\right)$. On the other hand, sensitivity analysis was adopted to evaluate the effect of a single study on the pooled ORs by removing each study in turn from the pooled analysis. Although the heterogeneity had not changed obviously, the $\mathrm{P}$ values for pooled ORs under allelic comparison, heterozygous comparison and dominant comparison were reversed when the study [24] led by Elshazli R was removed. Therefore, we deleted this study and recalculated the relevant ORs and 95\%CIs to harvest a stable and credible outcome (Figure 3). The funnel plots were used to investigate publication bias and the outlines of the funnel plots appear to be symmetrical (Figure 5). For rs 231775 
SNP, the asymmetry of the funnel plot was attributed to Zhou et al.'s study [45] which was published in Chinese. HWE estimation indicated that allele or genotype frequencies were deviant from HWE in control group in the Liu et al.'s, Gonzalez-Escribano et al.'s and Sameem et al.'s studies [25, 29, 38], but the results of synthesis analysis were not substantially inversed. Hence, we didn't remove these studies from the meta-analysis.
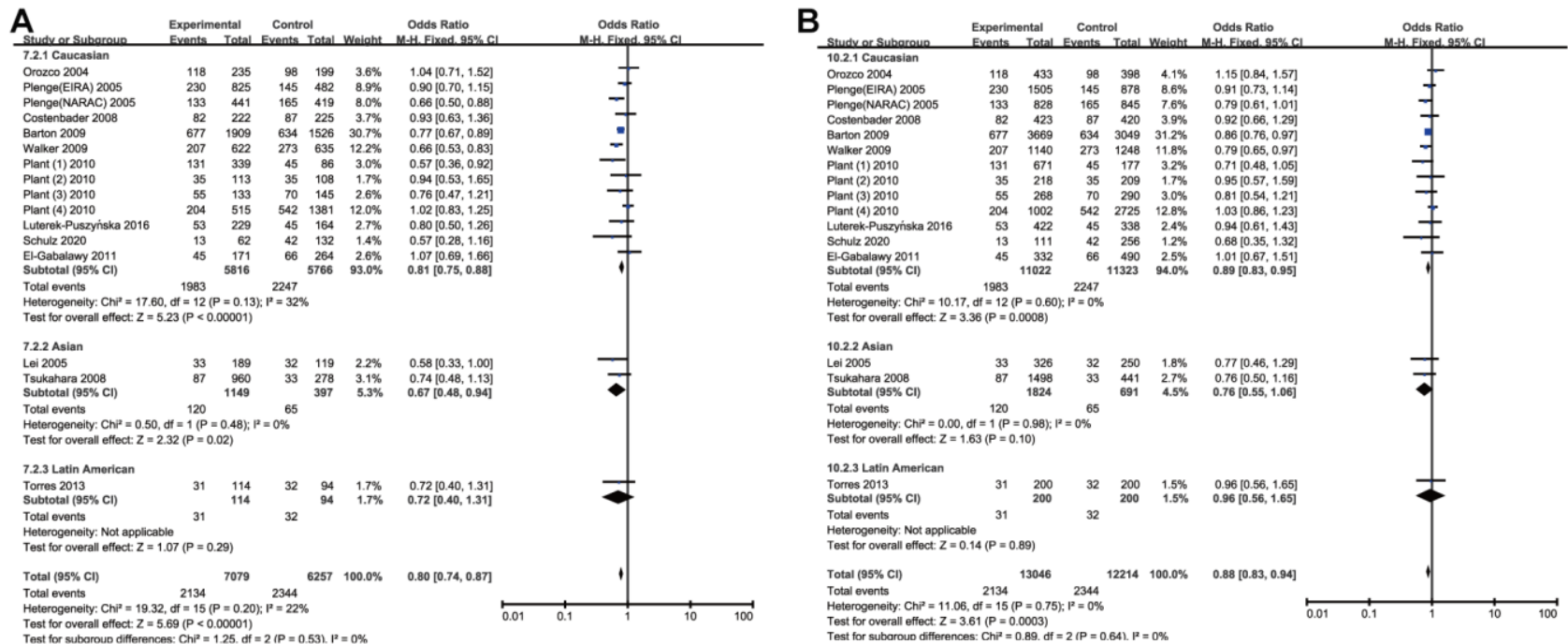

Figure 2. Forest plot of the association between rs308724 polymorphism and RA risk under the homozygous (A) and recessive model (B).
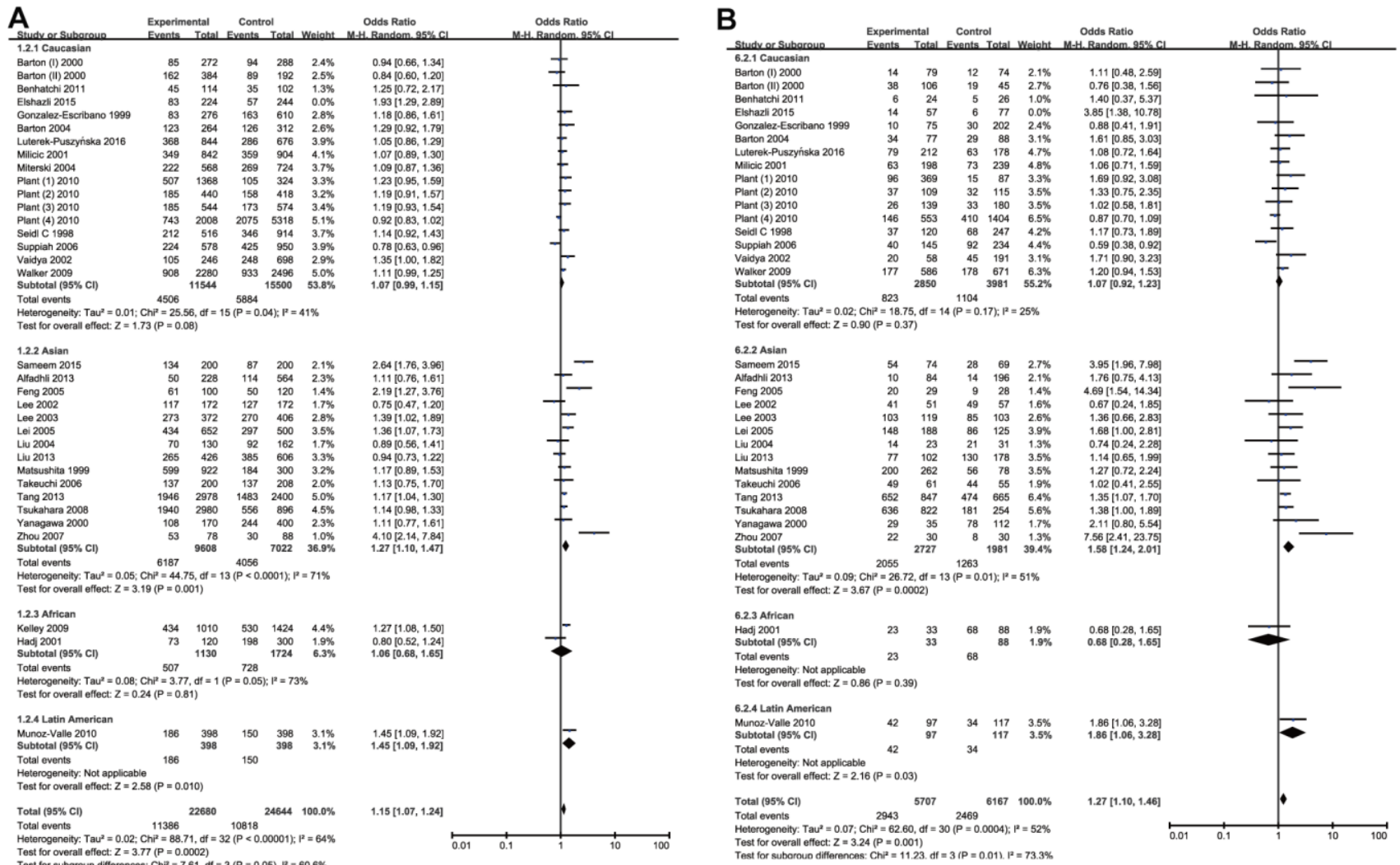

Figure 3. Forest plot of the association between rs231775 polymorphism and RA risk under the allelic model with Elshazli R et al.'s study excluded (A) and homozygous model (B). 


\section{DISCUSSION}

To our knowledge, this was the first meta-analysis to investigate the association between the three most-often SNPs of CTLA-4 and RA susceptibility. From the data integration of 66 studies in 21681 cases and 23457 controls, we found that the rs3087243 SNP decreased the risk of RA risk in Caucasians and Asians, the rs231775 SNP of CTLA-4 increased RA risk in Asians but not in Caucasians and Africans, and the rs5742909 SNP was not significantly associated with RA risk in both Caucasians and Africans.

The CTLA-4 gene, located on chromosome 2q33, encodes a 223 amino acid receptor protein on $\mathrm{T}$ cell surface which is responsible for $\mathrm{T}$ cell immune
A

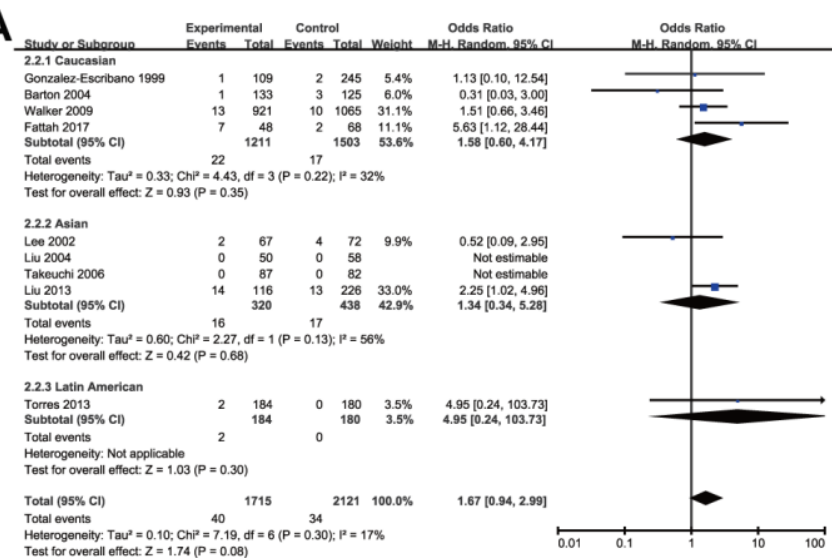

B

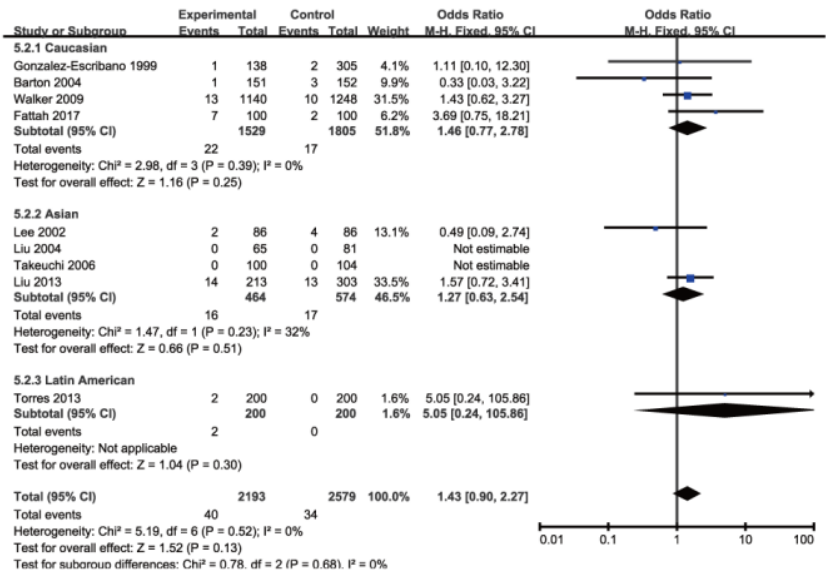

Figure 4. Forest plot of the association between rs574299 polymorphism and RA risk under the homozygous (A) and recessive model (B).
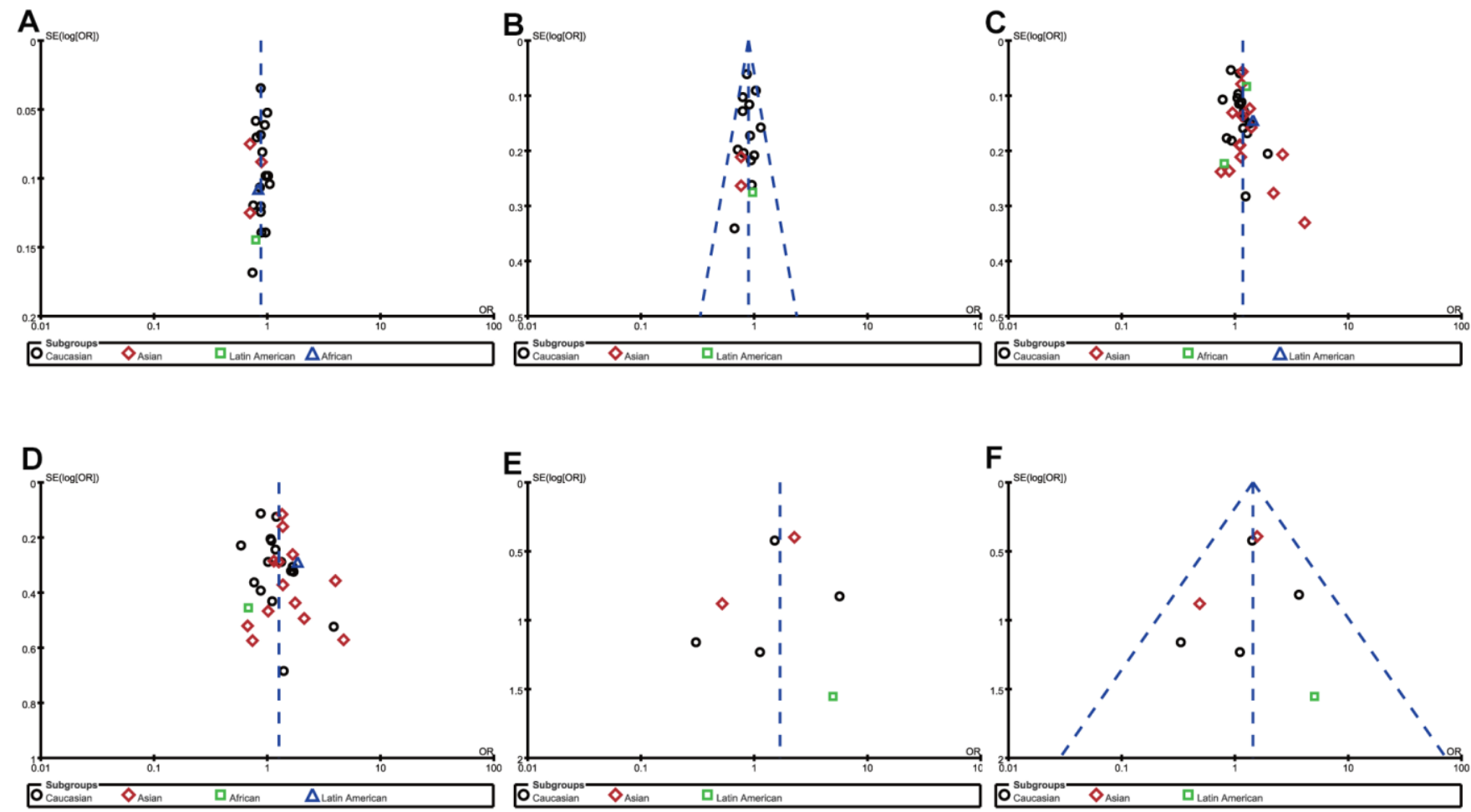

Figure 5. Funnel plot of the association between RA risk and rs308724 polymorphism under the allelic (A) and recessive model (B), rs231775 polymorphism under the allelic (C) and homozygous model (D), and rs574299 polymorphism under the homozygous (E) and recessive model (F). 
regulation. As an antagonist of the costimulatory receptor $\mathrm{CD} 28$ which binds the same ligand $\mathrm{B} 7$ as CTLA-4, CTLA-4 with higher affinity transmits an inhibitory signal and subsequently plays a suppressive role in regulating T-cell activation [57], which suggests it is involved in the pathological processes of many autoimmune disorders [12-15]. It is widely believed that RA is a T cell-mediated autoimmune disease [58], of which the chronic inflammation and damage of the joints are typical [1]. Although a great many genes whose protein products are critical to $\mathrm{T}$ cell function don't have genetic associations with RA, the effect of CTLA-4 on RA pathogenesis has attracted growing attentions.

Previous research had found that serum levels of soluble CTLA-4 were increased in RA patients and had a positive correlation with Disease Activity Score in RA patients and even proposed that serum levels of CTLA4 could serve as a new marker of RA disease activity $[59,60]$. Besides, function experiments in vivo indicated that gene delivery of CTLA4 by intra-articular injection could alleviate experimental arthritis [61]. Furthermore, CTLA-4Ig administration on RA synovial macrophages and $\mathrm{T}$ helper cells downregulated the production of proinflammatory cytokines, and these evidences suggested that CTLA- 4 could be a treatment target for RA [62, 63]. In fact, blockade of CTLA-4 by CTLA-4Ig had been successfully applied to treatment for RA [64].

As we all know, the protein level, structure and function are determined in large part by gene. Apart from these function research, numerous studies on correlation between CTLA-4 and RA risk from gene level also had been conducted to investigate genetic factors $[8,9,13-$ 15, 17-20, 24-56]. However, the results were inconsistent or contrary likely due to the various ethnic background, disparate geographic environment, limited sample size, insufficient data and so on. Thus, it was urgently necessary to perform a comprehensive up-todate meta-analysis as an effective methodology to draw an overall objective appraisal on the association between $C T L A-4$ polymorphism and RA susceptibility.

In the present meta-analysis, we extracted 66 studies with 21681 cases and 23457 controls to inspect the correlation between three most-often SNPs in the CTLA-4 gene and the risk of RA. There were 22 studies with 16394 cases and 17453 controls for rs3087243 SNP, 34 studies with 11452 cases and 12444 controls for rs231775 SNP, and 10 studies with 2477 cases and 2941 controls for rs5742909 SNP. For rs3087243 polymorphism, our findings demonstrated a decreased susceptibility of RA both in total and in Caucasians in any gene mode. In total, carriers with allele $A$ reduced an approximate $13 \%$ risk of RA than ones with allele $G$ and genotype $A A$ reduced $20 \%$ or so than genotype $G G$. Moreover, a decreased susceptibility of RA was respectively also found among Asians in the allele and homozygote comparison and among Latin Americans in the heterozygote and dominant comparison. However, only one study was included in Latin Americans and Asians so it needed to enlarge sample size to further research. For rs231775 polymorphism, significant association did exist among the whole population in all genetic models except recessive model: compared with allele $A$ and genotype $A A$, allele $G$ and genotype $G G$ and $G A$ respectively was associated with an increased risk of RA. The same association was observed in Asians and Latin Americans in the subgroup analysis. On the contrary, no significant association between rs 231775 SNP and RA risk could be detected in Caucasians and Africans using any gene model after excluding the Elshazli R's study [24] with the apparent heterogeneity. Here, it should be noted that only one or two case-control study was included in Africans and Latin Americans, so the conclusions were not particularly convincing. For rs5742909 polymorphism, no significant association between this locus polymorphism and RA risk was observed among any population in any model. Although the heterogeneity existed in some genetic model, but no obvious change had happened in heterogeneity and $P$ value for the pooled ORs when each study was individually removed by sensitivity analysis.

With regard to the diverse results of the same SNP on different populations, it might be attributed to clinical and genetic real heterogeneity of RA, interaction of genetic background and region environment, and even lack of vigorous statistical power. Besides, it was noteworthy that one important factor for the diverse and disparate results was linkage disequilibrium (LD). These CTLA-4 SNPs might be not definitely the causative alleles, but they were likely to be in LD with the causative alleles which were yet unidentified. And, LD was different between ethnic and racial groups.

It should be pointed out that previous several metaanalyses have summarized the effect of CTLA-4 polymorphism on RA risk [21-23, 65]. But a few points need to be taken notice. On one hand, the previous conclusions were discordant as the following: the conclusion of Li's (2014) study [65] on the association of rs231775 SNP of CTLA-4 with RA was contrary to the others; the genetic models which indicated significant association were diverse in these analyses. These differences were mainly originated from divergent diagnostic criteria, limited number of studies and sample sizes. On the other hand, all these metaanalyses focused on only one of the three well-studied loci except Li's study [23] on two. As we all know, the 
expression and function of the protein are determined by the whole gene. Therefore, it is of great necessity to investigate simultaneously the effect of all the 3 SNPs on RA risk to obtain an overall evaluation. Besides, the number of included studies in previous meta-analyses was small. Some original association studies $[9,15,24$ 27] have emerged in the past few years and they can be incorporated. Taking these points into considerations, we updated the meta-analysis to achieve a more valid and comprehensive estimation on the association of CTLA-4 gene and RA susceptibility.

However, some limitations of our study should be acknowledged. Firstly, the small sample size in some studies and the limited studies for some stratified analysis were not sufficient enough to detect the relationship. Especially, the results of populations including only one study should be interpreted with caution. Secondly, we only investigated the role of three loci polymorphisms. As CTLA-4 gene had various SNPs, the function of protein CTLA-4 depended on the whole gene and RA was a multigene susceptibility disease, more SNPs of CTLA-4 should be included. Thirdly, certain degree of heterogeneity still existed in rs5742909 polymorphism and some genetic models. Although the elimination of each single study did not distinctly alter the $P$ value, the results must still be treated cautiously. Fourthly, inadequate raw data in some studies result in the inability to calculate the number of the genotypes and perform stratified analysis by age, gender and autoantibody status such as RF etc. As a consequence, any potential gene-environment and gene-gene interactions could not be accessed.

In conclusion, this meta-analysis suggested that rs3087243 polymorphisms were corelated with a reduced RA risk in both Asian and Caucasian populations, rs231775 polymorphisms was associated with an increased risk of RA in Asians, and rs5742909 polymorphism had no significant association with RA risk. Larger-scale studies of populations with different ethnicities are encouraged to validate the role CTLA-4 played in the pathogenesis of RA.

\section{MATERIALS AND METHODS}

This meta-analysis was performed according to the Preferred Reporting Items for Systematic Reviews and Meta-Analyses (PRISMA) guidelines [66].

\section{Search strategy}

From the databases PubMed, EMBASE, Web of Science and, the China National Knowledge Infrastructure (CNKI) and Wan Fang data, a comprehensive systematic literature retrieval was conducted to derive all relevant studies published before 10 October, 2020 (the search was constantly updated to submission). The following terms as Medical Subject Heading and free words were applied: "CTLA-4 or cytotoxic T lymphocyte antigen-4" and "single nucleotide polymorphism or polymorphism or variant or variation" and "rheumatoid arthritis or RA". The bibliographic lists of included studies were also browsed for potential related studies. There were no restrictions on language and publication date in this study.

\section{Inclusion and exclusion criteria}

The current meta-analysis used the following inclusion criteria to screen available literatures: 1) case-control study; 2) evaluation of the associations between CTLA4 (rs3087243, rs231775 and rs5742909)polymorphism and RA risk; 3) with sufficient data for extract odds ratios (ORs) and 95\% confidence intervals(CIs); (4) with reported allele or genotype numbers or frequencies in cases and control group; 5) with a clear diagnostic criteria. Accordingly, we excluded meaninglessness literatures if they had the following trait: 1) case report, comment, animal studies and conference abstracts; 2) with no detailed allele or genotype data; 3 ) duplications or no controls.

\section{Data extraction and assess of quality}

Two independent investigators respectively conducted a literature search according to the above search strategy, screened each article based on the predesigned inclusion and exclusion criteria, and extracted data from these eligible studies. It would be settled by discussion with the third party when the disagreement between investigators occurred. The following information was collected from every paper: 1) first author's surname, 2) the year of publication, 3) country or region of origin, 4) ethnicity, 5) total numbers of cases and controls, 6) genotype method, 7) diagnostic criteria, 8) polymorphism locus, 9) allele distribution or/and genotype distribution.

The methodological quality of included studies was accessed in light of the Newcastle-Ottawa Scale (NOS) for the evaluation of observational studies [67]. In brief, three broad perspectives were evaluated using the Star system (http://www.ohri.ca/programs/clinical epidemiology/oxford.asp). Any divergence between two investigators was solved by discussion until agreement was reached.

\section{Statistical analysis}

The strength of association of rs231775, rs5742909 and rs3087243 SNPs with RA risk was appraised via 
estimating ORs with their corresponding 95\% CIs. For each SNP, the pooled ORs were calculated individually for five gene models (allele model, homozygote model, heterozygote model, dominant model and recessive model). The $\mathrm{Z}$ test was used to evaluate the significance of the pooled ORs. $p<0.05$ was judged as statistically significant difference. Statistical Heterogeneity between studies was assessed by Chi square and $I^{2}$ values which range from $0 \%$ to $100 \%$. $25 \%, 50 \%$, and $75 \%$ were regarded as respectively low, moderate, and high level $[68,69]$. The random -effect model was employed when the value of $I^{2}$ was more than $50 \%$. If not, the fixed effect model was employed. Hardy-Weinberg equilibrium (HWE) was tested in the control group for all studies by Chi-square test to judge whether the selection bias existed. Potential publication bias was examined by funnel plots. Besides, the current metaanalysis had carried out subgroup analyses by the racial descent to assess the effects of ethnic background.

The above statistical analyses were performed using Review Manager 5.3 software (Nordic Cochrane Centre, Cochrane Collaboration, Copenhagen). All the $\mathrm{P}$ values were 2 -sided and $\mathrm{P}<0.05$ signified statistically significance.

\section{Supplementary information}

Additional file 1: Supplementary Table 1. Quality assessment of included studies according to the Newcastle-Ottawa Scale.

\section{AUTHOR CONTRIBUTIONS}

J.X. and H. L. conceived and designed this study. C. Z., S.G, X. Y., Z. S. and S. L. performed the experiments. C. Z., S.G analyzed the data. C. Z. and H. L. draft the manuscript. X.S. and J.X. revised the paper. All authors have contributed to the final version and approved the final manuscript.

\section{CONFLICTS OF INTEREST}

The authors declare that they have no conflicts of interest.

\section{FUNDING}

This study is supported by National Natural Science Foundation of China, Nos.81772396.

\section{REFERENCES}

1. Smolen JS, Aletaha D, Mclnnes IB. Rheumatoid arthritis. Lancet. 2016; 388:2023-38. https://doi.org/10.1016/S0140-6736(16)30173-8 PMID:27156434
2. Minichiello E, Semerano L, Boissier MC. Time trends in the incidence, prevalence, and severity of rheumatoid arthritis: A systematic literature review. Joint Bone Spine. 2016; 83:625-30.

https://doi.org/10.1016/j.jbspin.2016.07.007 PMID:27616690

3. Cross M, Smith E, Hoy D, Carmona L, Wolfe F, Vos T, Williams B, Gabriel S, Lassere M, Johns N, Buchbinder $R$, Woolf A, March L. The global burden of rheumatoid arthritis: estimates from the global burden of disease 2010 study. Ann Rheum Dis. 2014; 73:1316-22. https://doi.org/10.1136/annrheumdis-2013-204627 PMID:24550173

4. MacGregor AJ, Snieder $H$, Rigby AS, Koskenvuo $M$, Kaprio J, Aho K, Silman AJ. Characterizing the quantitative genetic contribution to rheumatoid arthritis using data from twins. Arthritis Rheum. 2000; 43:30-37.

https://doi.org/10.1002/1529-0131(200001)43:1<30:: AID-ANR5>3.0.CO;2-B PMID:10643697

5. Okada Y, Wu D, Trynka G, Raj T, Terao C, Ikari K, Kochi Y, Ohmura K, Suzuki A, Yoshida S, Graham RR, Manoharan A, Ortmann W, et al, and RACl consortium, and GARNET consortium. Genetics of rheumatoid arthritis contributes to biology and drug discovery. Nature. 2014; 506:376-81.

https://doi.org/10.1038/nature12873 PMID:24390342

6. Kim K, Bang SY, Lee HS, Bae SC. Update on the genetic architecture of rheumatoid arthritis. Nat Rev Rheumatol. 2017; 13:13-24.

https://doi.org/10.1038/nrrheum.2016.176 PMID:27811914

7. Leng RX, Di DS, Ni J, Wu XX, Zhang LL, Wang XF, Liu RS, Huang $Q$, Fan YG, Pan HF, Wang $B$, Ye DQ. Identification of new susceptibility loci associated with rheumatoid arthritis. Ann Rheum Dis. 2020; 79: 1565-71.

https://doi.org/10.1136/annrheumdis-2020-217351 PMID:32868391

8. Costenbader $\mathrm{KH}$, Chang SC, De Vivo I, Plenge R, Karlson EW. Genetic polymorphisms in PTPN22, PADI-4, and CTLA-4 and risk for rheumatoid arthritis in two longitudinal cohort studies: evidence of geneenvironment interactions with heavy cigarette smoking. Arthritis Res Ther. 2008; 10:R52. https://doi.org/10.1186/ar2421 PMID:18462498

9. Luterek-Puszyńska K, Malinowski D, ParadowskaGorycka A, Safranow K, Pawlik A. CD28, CTLA-4 and CCL5 gene polymorphisms in patients with rheumatoid arthritis. Clin Rheumatol. 2017; 36:1129-35. https://doi.org/10.1007/s10067-016-3496-2 PMID:27988812 
10. Klocke K, Sakaguchi S, Holmdahl R, Wing K. Induction of autoimmune disease by deletion of CTLA-4 in mice in adulthood. Proc Natl Acad Sci USA. 2016; 113:E2383-92.

https://doi.org/10.1073/pnas.1603892113

PMID:27071130

11. Sansom DM. IMMUNOLOGY. Moving CTLA-4 from the trash to recycling. Science. 2015; 349:377-78.

https://doi.org/10.1126/science.aac7888

PMID:26206917

12. Ueda H, Howson JM, Esposito L, Heward J, Snook H, Chamberlain G, Rainbow DB, Hunter KM, Smith AN, Di Genova G, Herr MH, Dahlman I, Payne F, et al. Association of the T-cell regulatory gene CTLA4 with susceptibility to autoimmune disease. Nature. 2003; 423:506-11.

https://doi.org/10.1038/nature01621

PMID: 12724780

13. Zhernakova A, Eerligh P, Barrera P, Wesoly JZ, Huizinga TW, Roep BO, Wijmenga C, Koeleman BP. CTLA4 is differentially associated with autoimmune diseases in the Dutch population. Hum Genet. 2005; 118:58-66. https://doi.org/10.1007/s00439-005-0006-z PMID:16025348

14. Walker EJ, Hirschfield GM, Xu C, Lu Y, Liu X, Lu Y, Coltescu C, Wang K, Newman WG, Bykerk V, Keystone $E C$, Mosher D, Amos $\mathrm{Cl}$, et al. CTLA4/ICOS gene variants and haplotypes are associated with rheumatoid arthritis and primary biliary cirrhosis in the Canadian population. Arthritis Rheum. 2009; 60:931-37.

https://doi.org/10.1002/art.24412

PMID:19333938

15. Schulz S, Zimmer P, Pütz N, Jurianz E, Schaller HG, Reichert S. rs2476601 in PTPN22 gene in rheumatoid arthritis and periodontitis-a possible interface? J Transl Med. 2020; 18:389.

https://doi.org/10.1186/s12967-020-02548-w

PMID:33059697

16. Yang J, McGovern A, Martin P, Duffus K, Ge X, Zarrineh $P$, Morris AP, Adamson A, Fraser $P$, Rattray $M$, Eyre $S$. Analysis of chromatin organization and gene expression in $T$ cells identifies functional genes for rheumatoid arthritis. Nat Commun. 2020; 11:4402.

https://doi.org/10.1038/s41467-020-18180-7

PMID:32879318

17. Barton A, Myerscough A, John S, Gonzalez-Gay M, Ollier W, Worthington J. A single nucleotide polymorphism in exon 1 of cytotoxic T-lymphocyteassociated-4 (CTLA-4) is not associated with rheumatoid arthritis. Rheumatology (Oxford). 2000; 39:63-66.

https://doi.org/10.1093/rheumatology/39.1.63

PMID: $\underline{10662875}$
18. Plenge RM, Padyukov L, Remmers EF, Purcell S, Lee AT, Karlson EW, Wolfe F, Kastner DL, Alfredsson L, Altshuler D, Gregersen PK, Klareskog L, Rioux JD. Replication of putative candidate-gene associations with rheumatoid arthritis in $>4,000$ samples from North America and Sweden: association of susceptibility with PTPN22, CTLA4, and PADI4. Am J Hum Genet. 2005; 77:1044-60. https://doi.org/10.1086/498651 PMID:16380915

19. Plant D, Flynn E, Mbarek $H$, Dieudé $P$, Cornelis $F$, Arlestig L, Dahlqvist SR, Goulielmos G, Boumpas DT, Sidiropoulos P, Johansen JS, Ørnbjerg LM, Hetland ML, et al. Investigation of potential non-HLA rheumatoid arthritis susceptibility loci in a European cohort increases the evidence for nine markers. Ann Rheum Dis. 2010; 69:1548-53.

https://doi.org/10.1136/ard.2009.121020 PMID:20498205

20. Liu CP, Jiang JA, Wang $T$, Liu XM, Gao L, Zhu RR, Shen $Y$, $\mathrm{Wu} \mathrm{M}, \mathrm{Xu} \mathrm{T}$, Zhang XG. CTLA-4 and CD86 genetic variants and haplotypes in patients with rheumatoid arthritis in southeastern China. Genet Mol Res. 2013; 12:1373-82. https://doi.org/10.4238/2013.April.25.8 PMID:23661460

21. Han S, Li Y, Mao Y, Xie Y. Meta-analysis of the association of CTLA-4 exon-1 +49A/G polymorphism with rheumatoid arthritis. Hum Genet. 2005; 118: 123-32.

https://doi.org/10.1007/s00439-005-0033-9 PMID:16133179

22. Lee YH, Bae SC, Choi SJ, Ji JD, Song GG. Association between the CTLA-4 +49 A/G polymorphism and susceptibility to rheumatoid arthritis: a meta-analysis. Mol Biol Rep. 2012; 39:5599-605. https://doi.org/10.1007/s11033-011-1364-3 PMID:22179750

23. Li X, Zhang C, Zhang J, Zhang Y, Wu Z, Yang L, Xiang Z, Qi Z, Zhang X, Xiao X. Polymorphisms in the CTLA-4 gene and rheumatoid arthritis susceptibility: a metaanalysis. J Clin Immunol. 2012; 32:530-39.

https://doi.org/10.1007/s10875-012-9650-y PMID:22354566

24. Elshazli R, Settin A, Salama A. Cytotoxic T lymphocyte associated antigen-4 (CTLA-4) +49 A>G gene polymorphism in Egyptian cases with rheumatoid arthritis. Gene. 2015; 558:103-07.

https://doi.org/10.1016/j.gene.2014.12.046 PMID:25542810

25. Sameem M, Rani A, Bashir R, Riaz N, Batool SA, Irfan S, Arshad M, Nawaz SK. CTLA-4+49 Polymorphism and Susceptibility to Rheumatoid Arthritis in Pakistani Population. Pak J Zool. 2015; 47:1731-37. 
26. Vernerova L, Spoutil F, Vlcek M, Krskova K, Penesova A, Meskova M, Marko A, Raslova K, Vohnout B, Rovensky J, Killinger Z, Jochmanova I, Lazurova I, et al. A Combination of CD28 (rs1980422) and IRF5 (rs10488631) Polymorphisms Is Associated with Seropositivity in Rheumatoid Arthritis: A Case Control Study. PLoS One. 2016; 11:e0153316.

https://doi.org/10.1371/journal.pone.0153316 PMID:27092776

27. Fattah SA, Ghattas MH, Saleh SM, Abo-Elmatty DM. Cytotoxic T-lymphocyte-associated protein 4 gene polymorphism is related to rheumatoid arthritis in Egyptian population. Arch Physiol Biochem. 2017; 123:50-53.

https://doi.org/10.1080/13813455.2016.1230135 PMID:27808571

28. Seidl C, Donner H, Fischer B, Usadel KH, Seifried E, Kaltwasser JP, Badenhoop K. CTLA4 codon 17 dimorphism in patients with rheumatoid arthritis. Tissue Antigens. 1998; 51:62-66.

https://doi.org/10.1111/j.1399-0039.1998.tb02947.x PMID: 9459504

29. Gonzalez-Escribano MF, Rodriguez R, Valenzuela $A$, Garcia A, Garcia-Lozano JR, Nuñez-Roldan A. CTLA4 polymorphisms in Spanish patients with rheumatoid arthritis. Tissue Antigens. 1999; 53:296-300.

https://doi.org/10.1034/i.1399-0039.1999.530311.x PMID:10203024

30. Matsushita M, Tsuchiya N, Shiota M, Komata $T$, Matsuta K, Zama K, Oka T, Juji T, Yamane A, Tokunaga K. Lack of a strong association of CTLA-4 exon 1 polymorphism with the susceptibility to rheumatoid arthritis and systemic lupus erythematosus in Japanese: an association study using a novel variation screening method. Tissue Antigens. 1999; 54:578-84. https://doi.org/10.1034/j.1399-0039.1999.540607.x PMID:10674972

31. Yanagawa T, Gomi K, Nakao El, Inada S. CTLA-4 gene polymorphism in Japanese patients with rheumatoid arthritis. J Rheumatol. 2000; 27:2740-42.

PMID: 11128657

32. Hadj Kacem H, Kaddour N, Adyel FZ, Bahloul Z, Ayadi H. HLA-DQB1 CAR1/CAR2, TNFa IR2/IR4 and CTLA-4 polymorphisms in Tunisian patients with rheumatoid arthritis and Sjögren's syndrome. Rheumatology (Oxford). 2001; 40:1370-74.

https://doi.org/10.1093/rheumatology/40.12.1370

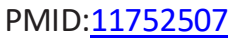

33. Milicic A, Brown MA, Wordsworth BP. Polymorphism in codon 17 of the CTLA-4 gene $(+49 A / G)$ is not associated with susceptibility to rheumatoid arthritis in British Caucasians. Tissue Antigens. 2001; 58:50-54. https://doi.org/10.1034/j.1399-0039.2001.580110.x PMID: 11580858

34. Lee YH, Choi SJ, Ji JD, Song GG. No association of polymorphisms of the CTLA-4 exon $1(+49)$ and promoter(-318) genes with rheumatoid arthritis in the Korean population. Scand J Rheumatol. 2002; 31:266-70.

https://doi.org/10.1080/030097402760375142 PMID: 12455815

35. Vaidya B, Pearce SH, Charlton S, Marshall N, Rowan $A D$, Griffiths ID, Kendall-Taylor P, Cawston TE, YoungMin S. An association between the CTLA4 exon 1 polymorphism and early rheumatoid arthritis with autoimmune endocrinopathies. Rheumatology (Oxford). 2002; 41:180-83.

https://doi.org/10.1093/rheumatology/41.2.180 PMID:11886967

36. Lee CS, Lee YJ, Liu HF, Su CH, Chang SC, Wang BR, Chen $T L$, Liu TL. Association of CTLA4 gene A-G polymorphism with rheumatoid arthritis in Chinese. Clin Rheumatol. 2003; 22:221-24. https://doi.org/10.1007/s10067-003-0720-7 PMID:14505215

37. Barton A, Jury F, Eyre S, Bowes J, Hinks A, Ward D, Worthington J. Haplotype analysis in simplex families and novel analytic approaches in a case-control cohort reveal no evidence of association of the CTLA-4 gene with rheumatoid arthritis. Arthritis Rheum. 2004; 50:748-52.

https://doi.org/10.1002/art.20118

PMID:15022315

38. Liu MF, Wang CR, Chen PC, Lin TL. CTLA-4 gene polymorphism in promoter and exon-1 regions is not associated with Chinese patients with rheumatoid arthritis. Clin Rheumatol. 2004; 23:180-81.

https://doi.org/10.1007/s10067-003-0776-4 PMID:15045639

39. Miterski B, Drynda S, Böschow G, Klein W, Oppermann J, Kekow J, Epplen JT. Complex genetic predisposition in adult and juvenile rheumatoid arthritis. BMC Genet. 2004; 5:2.

https://doi.org/10.1186/1471-2156-5-2 PMID:15018649

40. Orozco G, Torres B, Núñez-Roldán A, GonzálezEscribano MF, Martín J. Cytotoxic T-lymphocyte antigen-4-CT60 polymorphism in rheumatoid arthritis. Tissue Antigens. 2004; 64:667-70.

https://doi.org/10.1111/j.1399-0039.2004.00318.x PMID:15546339

41. Lei C, Dongqing Z, Yeqing S, Oaks MK, Lishan C, Jianzhong J, Jie Q, Fang D, Ningli L, Xinghai H, Daming R. Association of the CTLA-4 gene with rheumatoid 
arthritis in Chinese Han population. Eur J Hum Genet. 2005; 13:823-28.

https://doi.org/10.1038/sj.ejhg.5201423

PMID:15841095

42. Feng ZL. [CTLA-4 gene $A / G(49)$ polymorphism association with SLE and RA and expression of soluble CTLA-4 in patients with SLE and with RA]. Shandong University. 2005.

43. Suppiah V, O’Doherty C, Heggarty S, Patterson CC, Rooney M, Vandenbroeck K. The CTLA4+49A/G and CT60 polymorphisms and chronic inflammatory arthropathies in Northern Ireland. Exp Mol Pathol. 2006; 80:141-46.

https://doi.org/10.1016/i.yexmp.2005.09.004 PMID:16248997

44. Takeuchi F, Kawasugi K, Mori M, Nakaue N, Kobayashi $\mathrm{N}$, Kuwata S, Murayama T, Matsuta K. The genetic contribution of CTLA-4 dimorphisms in promoter and exon 1 regions in Japanese patients with rheumatoid arthritis. Scand J Rheumatol. 2006; 35:154-55. https://doi.org/10.1080/03009740500407651 PMID:16641053

45. Zhou Y, Xiao LS. [Association of the polymorphism of CTLA-4 gene with systemic lupus erythematosus or rheumatoid arthitis in the Chinese population]. Immunol J. 2007; 3:349-50.

46. Tsukahara S, Iwamoto $\mathrm{T}$, Ikari $\mathrm{K}$, Inoue $\mathrm{E}$, Tomatsu $\mathrm{T}$, Hara M, Yamanaka H, Kamatani N, Momohara S. CTLA4 CT60 polymorphism is not an independent genetic risk marker of rheumatoid arthritis in a Japanese population. Ann Rheum Dis. 2008; 67:428-29.

https://doi.org/10.1136/ard.2007.079186 PMID:18292106

47. Barton A, Eyre S, Ke X, Hinks A, Bowes J, Flynn E, Martin P, Wilson AG, Morgan AW, Emery P, Steer S, Hocking LJ, Reid DM, et al, and YEAR Consortium, and BIRAC Consortium. Identification of AF4/FMR2 family, member 3 (AFF3) as a novel rheumatoid arthritis susceptibility locus and confirmation of two further pan-autoimmune susceptibility genes. Hum Mol Genet. 2009; 18:2518-22. https://doi.org/10.1093/hmg/ddp177 PMID:19359276

48. Daha NA, Kurreeman FA, Marques RB, StoekenRijsbergen G, Verduijn W, Huizinga TW, Toes RE. Confirmation of STAT4, IL2/IL21, and CTLA4 polymorphisms in rheumatoid arthritis. Arthritis Rheum. 2009; 60:1255-60.

https://doi.org/10.1002/art.24503 PMID:19404967

49. Kelley JM, Hughes LB, Faggard JD, Danila MI, Crawford $\mathrm{MH}$, Edberg $\mathrm{Y}$, Padilla MA, Tiwari HK, Westfall AO, Alarcón GS, Conn DL, Jonas BL, Callahan LF, et al. An African ancestry-specific allele of CTLA4 confers protection against rheumatoid arthritis in African Americans. PLoS Genet. 2009; 5:e1000424. https://doi.org/10.1371/journal.pgen.1000424 PMID:19300490. Retraction in: PLoS Genet. 2009; 5. https://doi.org/10.1371/annotation/80bd7285-9d2d403a-8e6f-9c375bf977ca PMID:20020059

50. Muñoz-Valle JF, Valle Y, Padilla-Gutiérrez JR, ParraRojas I, Rangel-Villalobos $H$, Vázquez del Mercado $M$, Ledezma-Lozano IY, Villafan-Bernal JR, ArmendárizBorunda J, Pereira-Suárez AL. The +49A>G CTLA-4 polymorphism is associated with rheumatoid arthritis in Mexican population. Clin Chim Acta. 2010; 411: 725-28.

https://doi.org/10.1016/i.cca.2010.02.001 PMID:20138855

51. Benhatchi $\mathrm{K}$, Jochmanová I, Habalová V, Wagnerová $\mathrm{H}$, Lazúrová I. CTLA4 exon1 A49G polymorphism in Slovak patients with rheumatoid arthritis and Hashimoto thyroiditis-results and the review of the literature. Clin Rheumatol. 2011; 30:1319-24.

https://doi.org/10.1007/s10067-011-1752-z PMID:21503616

52. Danoy $P$, Wei $M$, Johanna $H$, Jiang $L$, He $D$, Sun $L$, Zeng $X$, Visscher PM, Brown MA, Xu H. Association of variants in MMEL1 and CTLA4 with rheumatoid arthritis in the Han Chinese population. Ann Rheum Dis. 2011; 70:1793-97.

https://doi.org/10.1136/ard.2010.144576

PMID:21784728

53. El-Gabalawy HS, Robinson DB, Daha NA, Oen KG, Smolik I, Elias B, Hart D, Bernstein CN, Sun Y, Lu Y, Houwing-Duistermaat JJ, Siminovitch KA. Non-HLA genes modulate the risk of rheumatoid arthritis associated with HLA-DRB1 in a susceptible North American Native population. Genes Immun. 2011; 12:568-74. https://doi.org/10.1038/gene.2011.30 PMID:21614018

54. AlFadhli S. Overexpression and secretion of the soluble CTLA-4 splice variant in various autoimmune diseases and in cases with overlapping autoimmunity. Genet Test Mol Biomarkers. 2013; 17:336-41. https://doi.org/10.1089/gtmb.2012.0391 PMID:23448385

55. Tang MJ, Zhou ZB. Association of the CTLA-4 +49A/G polymorphism with rheumatoid arthritis in Chinese Han population. Mol Biol Rep. 2013; 40:2627-31.

https://doi.org/10.1007/s11033-012-2349-6 PMID:23264071

56. Torres-Carrillo N, Ontiveros-Mercado H, Torres-Carrillo NM, Parra-Rojas I, Rangel-Villalobos H, RamírezDueñas MG, Gutiérrez-Ureña SR, Valle Y, Muñoz-Valle JF. The $-319 C /+49 G / C T 60 G$ haplotype of CTLA-4 gene confers susceptibility to rheumatoid arthritis in 
Mexican population. Cell Biochem Biophys. 2013; 67:1217-28.

https://doi.org/10.1007/s12013-013-9640-6

PMID:23703660

57. Walker LS, Sansom DM. Confusing signals: recent progress in CTLA-4 biology. Trends Immunol. 2015; 36:63-70.

https://doi.org/10.1016/j.it.2014.12.001

PMID:25582039

58. Cope AP, Schulze-Koops H, Aringer M. The central role of T cells in rheumatoid arthritis. Clin Exp Rheumatol. 2007 (Suppl 46); 25:S4-11.

PMID: 17977483

59. Cao J, Zou L, Luo P, Chen P, Zhang L. Increased production of circulating soluble co-stimulatory molecules CTLA-4, CD28 and CD80 in patients with rheumatoid arthritis. Int Immunopharmacol. 2012; 14:585-92.

https://doi.org/10.1016/j.intimp.2012.08.004

PMID:22917707

60. Choi IS, Yoo HS, Collisson EW. Evaluation of expression patterns of feline CD28 and CTLA-4 in feline immunodeficiency virus (FIV)-infected and FIV antigeninduced PBMC. J Vet Sci. 2000; 1:97-103.

https://doi.org/10.4142/jvs.2000.1.2.97

PMID:14614304

61. Zhang W, Wang F, Wang B, Zhang J, Yu JY. Intraarticular gene delivery of CTLA4-FasL suppresses experimental arthritis. Int Immunol. 2012; 24:379-88. https://doi.org/10.1093/intimm/dxs041

PMID:22354915

62. Cutolo $M$, Soldano $S$, Montagna $P$, Sulli $A$, Seriolo $B$, Villaggio B, Triolo P, Clerico P, Felli L, Brizzolara R. CTLA4-Ig interacts with cultured synovial macrophages from rheumatoid arthritis patients and downregulates cytokine production. Arthritis Res Ther. 2009; 11:R176. https://doi.org/10.1186/ar2865

PMID:19930661
63. Körmendy D, Hoff $H$, Hoff $P$, Bröker BM, Burmester GR, Brunner-Weinzierl MC. Impact of the CTLA-4/CD28 axis on the processes of joint inflammation in rheumatoid arthritis. Arthritis Rheum. 2013; 65:81-87.

https://doi.org/10.1002/art.37714 PMID:23045162

64. Kremer JM, Westhovens R, Leon M, Di Giorgio E, Alten $R$, Steinfeld $S$, Russell $A$, Dougados $M$, Emery $P$, Nuamah IF, Williams GR, Becker JC, Hagerty DT, Moreland LW. Treatment of rheumatoid arthritis by selective inhibition of T-cell activation with fusion protein CTLA4lg. N Engl J Med. 2003; 349:1907-15. https://doi.org/10.1056/NEJMoa035075 PMID:14614165

65. Li G, Shi F, Liu J, Li Y. The effect of CTLA-4 A49G polymorphism on rheumatoid arthritis risk: a metaanalysis. Diagn Pathol. 2014; 9:157. https://doi.org/10.1186/s13000-014-0157-0 PMID:25128482

66. Moher D, Liberati A, Tetzlaff J, Altman DG, and PRISMA Group. Preferred reporting items for systematic reviews and meta-analyses: the PRISMA statement. BMJ. 2009; 339:b2535.

https://doi.org/10.1136/bmj.b2535

PMID:19622551

67. Stang A. Critical evaluation of the Newcastle-Ottawa scale for the assessment of the quality of nonrandomized studies in meta-analyses. Eur J Epidemiol. 2010; 25:603-05. https://doi.org/10.1007/s10654-010-9491-z PMID:20652370

68. Higgins JP, Thompson SG. Quantifying heterogeneity in a meta-analysis. Stat Med. 2002; 21:1539-58. https://doi.org/10.1002/sim.1186 PMID:12111919

69. Higgins JP, Thompson SG, Deeks JJ, Altman DG. Measuring inconsistency in meta-analyses. BMJ. 2003; 327:557-60. https://doi.org/10.1136/bmj.327.7414.557 PMID: $\underline{12958120}$ 


\section{SUPPLEMENTARY MATERIALS}

\section{Supplementary Table}

Supplementary Table 1. Quality assessment of included studies according to the Newcastle-Ottawa Scale.

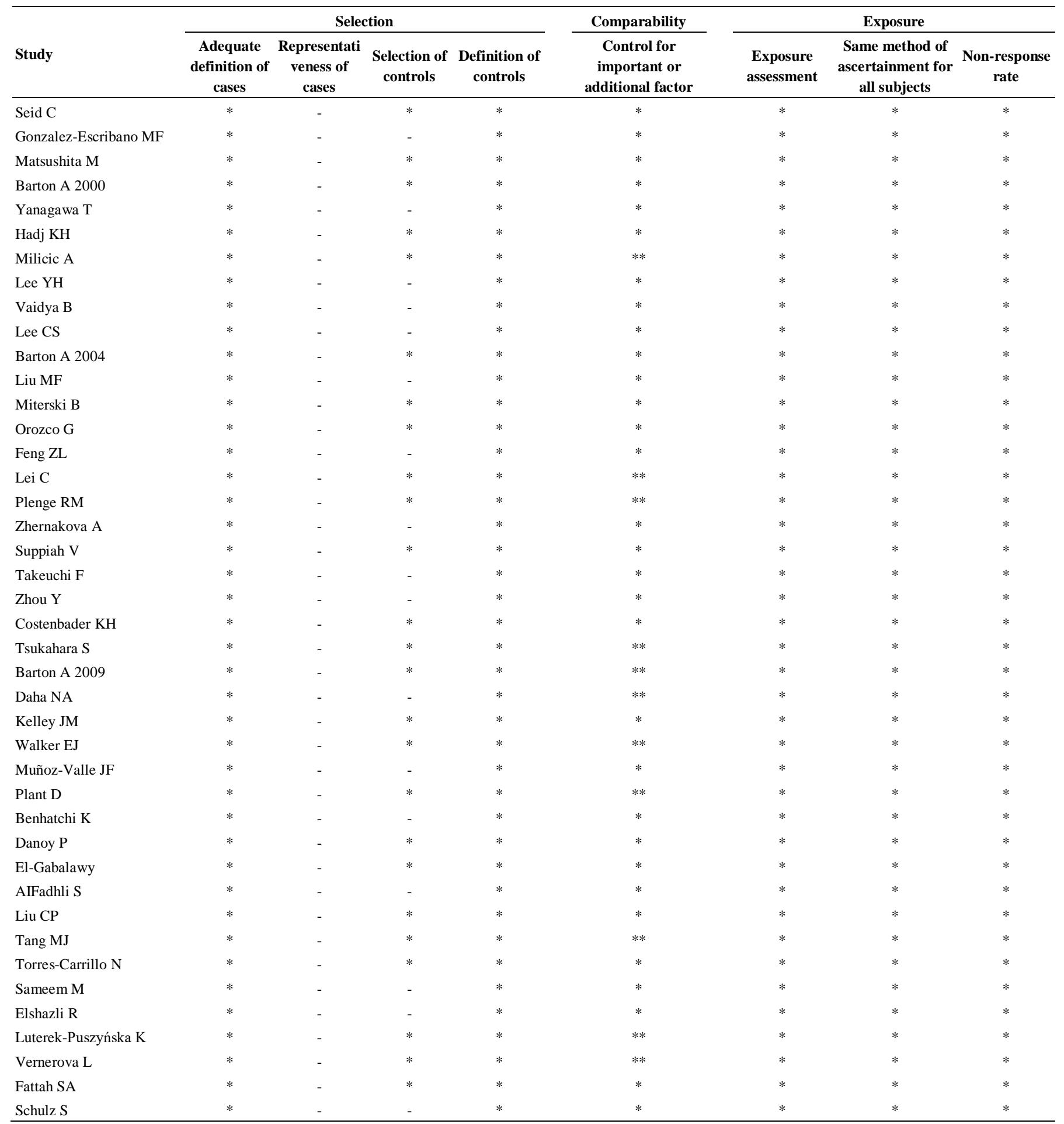

A study could be awarded a one or zero star for every item except for the item "Control for important factor or additional factor". 